\title{
The rice Aux/IAA transcription factor gene OsIAA18 enhances salt and osmotic tolerance in Arabidopsis
}

\author{
G. LI ${ }^{1}$, Y.X. YE ${ }^{2}$, X.Q. REN ${ }^{2}$, M.Y. QI ${ }^{2}$, H.Y. ZHAO 2 , Q. ZHOU ${ }^{2}$, X.H. CHEN ${ }^{2}$, J. WANG ${ }^{2}$, \\ C.Y. YUAN ${ }^{1 *}$, and F.B. WANG ${ }^{2 *}$
}

\begin{abstract}
Huaiyin Institute of Agricultural Sciences of Xuhuai Region, Huai'an Jiangsu 223001, P.R. China ${ }^{1}$
School of Life Science and Food Engineering, Huaiyin Institute of Technology, Huai'an Jiangsu 223003, P.R. China ${ }^{2}$
\end{abstract}

\begin{abstract}
In plants, auxin/indoleacetic acid (Aux/IAA) proteins are transcriptional regulators, which regulate developmental process and responses to phytohormones and stress treatments. A previous study has shown that the rice Aux/IAA transcription factor gene OsIAA18 is induced by salt and osmotic stresses. However, little is known about the regulatory functions of this gene. In this study, the OSIAA18 gene was successfully cloned from rice. Subcellular localization analysis in onion epidermal cells indicated that OsIAA18 was localized to the nucleus. Expression analysis in yeast showed that the full length OsIAA18 exhibited transcriptional activation. Heterologous expression of OsIAA18 significantly enhanced salt and osmotic tolerance in transgenic Arabidopsis plants. Real-time quantitative PCR analysis showed that constitutive expression of OsIAA18 up-regulated genes involved in abscisic acid (ABA) biosynthesis, proline biosynthesis, stress responses, and reactive oxygen species scavenging under salt and osmotic stresses. Enzymatic analyses found that the transgenic plants had higher 9-cis-epoxycarotenoid dioxygenase, pyrroline-5-carboxylate synthase, superoxide dismutase, and peroxidase activities than wild-type plants under salt and osmotic stresses. In the transgenic plants, ABA and proline content significantly increased, whereas $\mathrm{H}_{2} \mathrm{O}_{2}$ and malondialdehyde content significantly decreased. In addition, the transgenic plants had also a lower electrolyte leakage and water loss rate. These overall results indicate that the OsIAA18 gene is involved in enhancing salt and osmotic tolerance in transgenic Arabidopsis plants. The OsIAA18 gene has a potential to be used to enhance the tolerance to abiotic stresses in other plant species.
\end{abstract}

Additional key words: abscisic acid, onion, Oryza sativa, MDA, POD, proline, ROS, SOD, yeast.

\section{Introduction}

Growth and development of crops worldwide have been adversely impacted by salt and osmotic stresses, which are becoming serious threats to agricultural efforts to provide and satisfy the needs of a rapidly growing human population (Munns and Tester 2008, Wang et al. 2016a-d). It has been reported that approximately $20 \%$ of the irrigated soils are suffering from salt stress (Zhao et al. 2013). Meanwhile, the problems of global water scarcity and worldwide climate change are threatening sustainable crop farming (Yang et al. 2010). Therefore, it is necessary to enhance salt and osmotic tolerance of crops. The studies indicate that some plants have evolved counter-mechanisms, such as growth and development regulation, detoxification, ion homeostasis, and osmotic adjustment, in order to adapt to abiotic stresses (including salinity, drought, and osmotic stress) (Bohnert et al. 1995, Zhu 2001, 2002).

Auxin plays a very important role in a wide variety of plant developmental and physiological processes (Friml 2003, Song et al. 2009). It has been shown that auxin signalling, recruiting specific transcription factors to regulate the expression of down-stream genes, performs the required responses (Vogler and Kuhlemeier 2003).

\footnotetext{
Submitted 10 December 2018, last revision 15 April 2019, accepted 23 April 2019.

Abbreviations: ABA - abscisic acid; Aux/IAA - auxin/indoleacetic acid; GFP - green fluorescent protein; Hyg - hygromycin; MDA - malondialdehyde; NCED - 9-cis-epoxycarotenoid dioxygenase; ORF - open reading frame; PEG - polyethylene glycol; POD peroxidase; P5CS - pyrroline-5-carboxylate synthase; ROS - reactive oxygen species; SOD - superoxide dismutase; WT - wild-type. Acknowledgments: This research was financially supported by the National Key Research and Development Program of China (2017YFD100400), the Key Research and Development Program of Huai'an City (HAN201805 and HAN201810), the Natural Science Fund for Colleges and Universities in Jiangsu Province of China (17KJB210001), and the Talent Introduction Research Project of Huaiyin Institute of Technology (Z301B16534). Feibing Wang, Gang Li, Yuxiu Ye, and Xuqin Ren have contributed equally to this work.

*Corresponding authors; e-mails: wangfeibing1986@163.com, hysdycy@163.com
} 
Auxin/indoleacetic acid (Aux/IAA) and auxin response factor (ARF) are two important protein families in developmental process and responses to phytohormones and stress treatments by controlling auxin-responsive transcription (Berleth et al. 2004, Hagen and Guilfoyle 2002, Liscum and Reed 2002, Song et al. 2009).

The Aux/IAA-encoding genes have been cloned and identified in mung bean (Yamamoto 1994), Arabidopsis (Reed 2001), and rice (Thakur et al. 2001, Nakamura et al. 2006). In rice, 31 Aux/IAA genes (OSIAA) was identified and their sequences were analyzed (Jain et al. 2006, Wang et al. 2007). The first rice OsIAA gene, named OsIAA13, was cloned by Thakur et al. $(2001,2005)$, while the first Aux/IAA protein OsIAA31 was characterized in rice by Nakamura et al. (2006). The rice plants overexpressing OsIAA31 show insensitivity to auxin and gravitropic stimuli and exhibit short leaf blades, reduced crown root formation, and abnormal leaf formation (Nakamura et al. 2006). OsIAA23 plays an important role in postembryonic maintenance of quiescent center (Jun et al. 2011). OsIAA13 is involved in lateral root initiation (Kitomi et al. 2012). OsIAA11 affects lateral root development (Zhu et al. 2012). Overexpression of OSIAA4 in rice induces morphological changes and reduces responsiveness to auxin (Song and $\mathrm{Xu}$ 2013). Song et al. (2009) found that most OsIAA genes are responsive to various abiotic stresses, indicating an interaction between plant growth and abiotic stress. However, the role of the OSIAA genes in enhancing tolerance to abiotic stresses has not been reported.

The OsIAA18 gene is induced by salt, osmotic, IAA, and abscisic acid (ABA) treatments in rice (Song et al. 2009). However, little work has been devoted to the regulatory functions of this gene. Therefore, we isolated the OsIAA18 gene (Genebank accession No. NP_001046357) from rice and estimated its roles in transgenic Arabidopsis. The aim was to confirm our hypothesis that heterologous expression of OSIAA18 can significantly enhance salt and osmotic tolerance in transgenic Arabidopsis.

\section{Materials and methods}

Plants: Rice (Oryza sativa L.) cultivar Nipponbare (Huaiyin Institute of Agricultural Sciences of Xuhuai Region, Huai'an, China) was employed for the OSIAA18 gene cloning in this study. One expressed sequence tag (EST) encoding OsIAA18 protein was selected from the cDNA-AFLP library for cloning this gene. Arabidopsis thaliana [ecotype Columbia-0, wild type (WT)] (Huaiyin Institute of Technology, Huai'an, China) was used as a model plant to identify the functions of this gene.

Cloning and sequence analysis of the rice $O s I A A 18$ gene: Total RNA was extracted from the leaves of cv. Nipponbare with the RNAprep Pure kit (Tiangen Biotech, Beijing, China). RNA samples were reversetranscribed according to the instructions of Quantscript reverse transcriptase kit (Tiangen Biotech). Based on the sequence of OSIAA18, we designed one gene-specific primer of reverse transcription PCR (RT-PCR) (Table 1
Suppl.) to obtain its full-length cDNA sequence. PCR was performed with an initial denaturation at $94{ }^{\circ} \mathrm{C}$ for $3 \mathrm{~min}$, followed by 35 cycles of $94{ }^{\circ} \mathrm{C}$ for $30 \mathrm{~s}, 55^{\circ} \mathrm{C}$ for $30 \mathrm{~s}$, $72{ }^{\circ} \mathrm{C}$ for $1 \mathrm{~min}$, and final extension at $72{ }^{\circ} \mathrm{C}$ for $10 \mathrm{~min}$. PCR products were separated on a $1.0 \%(\mathrm{~m} / \mathrm{v})$ agarose gel. Target DNA bands were recovered by gel extraction, then cloned into PMD19-T (TaKaRa, Beijing, China), and finally transformed into competent cells of Escherichia coli strain DH5 $\alpha$. White colonies were checked by PCR and the positive colonies were sequenced (Invitrogen, Beijing, China).

The full-length cDNA of OSIAA18 was analyzed by an online BLAST at the National Center for Biotechnology Information (NCBI) website (http://www.ncbi.nlm.nih. gov/, 06-10-2017). For the multiple sequence alignment analysis, the amino acid sequence of OSIAA18 and other $I A A$ homologs from different plant species retrieved from $N C B I$ were aligned using the DNAMAN software (Lynnon Biosoft, Quebec, Canada). The phylogenetic analysis was conducted with the MEGA4 software (http://www. megasoftware.net/). Theoretical molecular mass and isoelectronic point ( $\mathrm{pI}$ ) were calculated using ProtParam tool (http://web.expasy.org/protparam/). The conserved domain of OsIAA18 protein was scanned by InterProScan program (http://www.ebi.ac.uk/Tools/pfa/iprscan/). The nuclear localization signal of OsIAA18 protein was predicted by $c N L S$ Mapper program (http://nls-mapper. iab.keio.ac.jp/cgi-bin/NLS_Mapper_form.cgi).

Subcellular localization and transactivation assay of OsIAA18: Subcellular localization of OsIAA18 in onion (Allium cepa) epidermal cells was analyzed as described by Wang et al. (2016b). Transactivation assay of OsIAA18 in yeast (Saccharomyces cerevisiae) was conducted according to the method of Jiang et al. (2014). GUS activities were assayed by the fluorometric method (Jefferson et al. 1987).

Transformation of Arabidopsis with OSIAA18: The coding region of the synthesized OsIAA18 gene was cloned with terminal BamH I and Sac I restriction sites (Table 1 Suppl.) and then inserted into the same restriction sites in vector pCAMBIA1301 to create expression vector pCAMBIA1301-OsIAA18 under the control of CaMV $35 \mathrm{~S}$ promoter and NOS terminator of the expression box. This vector also contained $\beta$-glucuronidase (gusA) and hygromycin ( $h p t$ II) genes driven by a CaMV $35 \mathrm{~S}$ promoter, respectively. The recombinant vector was transformed into the Agrobacterium tumefaciens strain LBA4404 cells according to the methods described previously (Lou et al. 2007), and transgenic plants were produced according to methods described in another publication (Zhang et al. 2006). Transformants were selected based on their resistance to hygromycin (Hyg). Putative transformed seeds were germinated on agar-solidified Murashige and Skoog (1962; MS) medium containing $25 \mathrm{mg} \mathrm{dm}^{-3}$ Hyg. Positively transgenic seedlings were grown in pots containing a mixture of soil, Vermiculite, and humus $(1: 1: 1, v / v / v)$ to obtain $T_{2}$ and $T_{3}$ seeds. The incubation and growth conditions of Arabidopsis were the same as 
described previously (Zhang et al. 2006).

Analysis of transgenic plants by PCR: The presence of the OSIAA18 expression construct in positive plants was assessed by PCR analysis using specific primers (Table 1 Suppl.). DNA was extracted from Arabidopsis leaves according to the instructions of EasyPure plant genomic DNA kit (Transgen, Beijing, China). PCR amplifications were performed with an initial denaturation at 94 for $3 \mathrm{~min}$, followed by 35 cycles at $94{ }^{\circ} \mathrm{C}$ for $30 \mathrm{~s}, 55^{\circ} \mathrm{C}$ for $30 \mathrm{~s}, 72{ }^{\circ} \mathrm{C}$ for $1 \mathrm{~min}$, and final extension at $72{ }^{\circ} \mathrm{C}$ for $10 \mathrm{~min}$. PCR products were separated by electrophoresis on a $1.0 \%(\mathrm{~m} / \mathrm{v})$ agarose gel.

Reverse transcription PCR analysis: Total leaf RNA was extracted from the transgenic plants and WT using the RNAprep Pure plant kit (Tiangen Biotech). RNA samples were reverse-transcribed using Quantscript reverse transcriptase kit (Tiangen Biotech). The cDNA solution was used as templates for PCR amplification with specific primers of the OSIAA18 gene (Table 1 Suppl.). Arabidopsis AtActin gene (Genebank accession No. NM112764) was used as an internal control and amplified by the specific primers (Table 1 Suppl.) (Li et al. 2013). PCR amplifications were performed as described above, and the PCR products were separated by electrophoresis on a $1 \%$ agarose gel. The same results were obtained for three independent experiments.

Immunobot analysis: OsIAA18 antibody (full-length) was obtained from Beijing Protein Innovation. Total proteins were extracted from leaves of transgenic and WT plants in extraction buffer $[50 \mathrm{mM}$ Tris- $\mathrm{HCl}(\mathrm{pH}$ of 8.0$), 150 \mathrm{mM} \mathrm{KCl}, 1 \mathrm{mM}$ EDTA, $0.5 \%(\mathrm{~m} / \mathrm{v})$ Triton $X$-100, $1 \mathrm{mM}$ dithiothreitol (DTT), $1 \mathrm{mM}$ phenyl methyl sulfonyl fluoride (PMSF), and a $1 \times$ protease inhibitor cocktail tablet (Applygen Technologies, Beijing, China)]. The content of extracted protein was determined by bicinchoninic acid method (Gao et al. 2011a). Immunobot analysis was performed according to the method of Gao et al. (2011a). Fifty micrograms of protein were separated by dodecyl sodium sulfate-polyacrylamide gel electrophoresis [SDS-PAGE; $15 \%(\mathrm{~m} / \mathrm{v})$ separation gel and $5.4 \%$ stacking gel]. After electrophoresis, the protein on the gel was transferred to a nitrocellulose transfer membrane (Whatman, Braunschweig, Germany) by semidry electrophoretic transfer cell and immunostaining was performed as described by Wang and Fang (2002). The protein extracts were mixed with SDS-PAGE sample buffer and detected by immunoblotting using OsIAA18 antibody (Wang and Fang 2002).

Assay for salt and osmotic tolerance: In vitro assay for salt and osmotic tolerance was conducted as described by Liu et al. (2015b). Transgenic Arabidopsis $\mathrm{T}_{3}$ and WT seeds were sown on Murashige and Skoog (MS) medium with $200 \mathrm{mM} \mathrm{NaCl}$ and $25 \%(\mathrm{~m} / \mathrm{v})$ PEG6000 and grown at a temperature of $22{ }^{\circ} \mathrm{C}$, a 13 -h photoperiod and an irradiance of $54 \mu \mathrm{mol} \mathrm{m} \mathrm{m}^{-2} \mathrm{~s}^{-1}$ for 2 weeks. Then their root length and fresh mass were measured.
In vivo assay for salt and osmotic tolerance was based on the method of Wang et al. (2016b). Two-weekold transgenic Arabidopsis $\mathrm{T}_{3}$ and WT seedlings were grown in 6-cm diameter pots containing a mixture of soil, Vermiculite, and humus $(1: 1: 1, \mathrm{v} / \mathrm{v} / \mathrm{v})$ in a greenhouse, with nine plants per pot. All pots were irrigated sufficiently with half-Hoagland solution for 2 weeks and then each pot was irrigated with a $200 \mathrm{~cm}^{3}$ of $300 \mathrm{mM} \mathrm{NaCl}$ solution every $2 \mathrm{~d}$ for 4 weeks, or subjected to drought (without watering) for 6 weeks. After treatment, the survival rate of these plants was observed immediately. All treatments were performed in triplicate.

Expression analysis of $O S I A A 18$ and the related genes: The expressions of genes related to ABA biosynthesis, proline biosynthesis, stress responses, and ROS scavenging, were analyzed by real-time quantitative PCR (qPCR) as described Wang et al. (2016b). Transgenic or WT plants were grown in pots for 2 weeks under $200 \mathrm{mM}$ $\mathrm{NaCl}$ or for 4 weeks under $25 \%$ PEG. Specific primers designed from conserved regions of genes were listed in Table 1 Suppl. Arabidopsis AtActin gene was used as an internal control. Quantification of the gene expression was done with comparative $\mathrm{C}_{\mathrm{T}}$ method (Schmittgen and Livak 2008).

Analyses of 9-cis-epoxycarotenoid dioxygenase, pyrroline-5-carboxylate synthase, superoxide dismutase, and peroxidase activities: The activities of pyrroline-5-carboxylate synthase (P5CS), superoxide dismutase (SOD), and peroxidase (POD) in the transgenic plants and WT grown in pots and incubated for 4 weeks under optimum growth condition, for 2 weeks under $300 \mathrm{mM} \mathrm{NaCl}$, or for 4 weeks under $25 \%$ PEG were measured according to the methods of Hayzer and Leisinger (1980), Gao et al. (2011b), and Lurie et al. (1997), respectively. The 9-cis-epoxycarotenoid dioxygenase (NCED) activity was measured with Plant NCED ELISA kit (USCN Life Science, Shanghai, China). One unit (U) of enzyme activity is defined as the amount of enzyme that catalyzes conversion of one micromole of substrate per minute under the specified conditions of the assay method.

Measurements of the content of $\mathrm{ABA}$, proline, malondialdehyde, and $\mathrm{H}_{2} \mathrm{O}_{2}$, and electrolyte leakage: The content of ABA, proline, malondialdehyde (MDA), and $\mathrm{H}_{2} \mathrm{O}_{2}$ in the transgenic Arabidopsis plants and WT grown in pots and incubated for 4 weeks under optimum growth condition, for 2 weeks under $300 \mathrm{mM} \mathrm{NaCl}$ stress, or for 4 weeks under $25 \%$ PEG were measured according to the methods of Gao et al. (2011b) and Alexieva et al. (2001).

Electrolyte leakage rates in the transgenic and WT plants were determined according to Ben-Amor et al. (1999). Water loss rate was measured according the method of Liu et al. (2015b).

Statistical analysis: The experiments were repeated three times and the data presented are the means \pm standard errors (SEs). Where applicable, data were analyzed by 
the Student's $t$-test in a two-tailed analysis using SPSS v. 17.0. Values of $P<0.05$ or $<0.01$ were considered to be statistically significant.

\section{Results}

The OSIAA18 gene was cloned by RT-PCR. The ORF was $903 \mathrm{bp}$ in size and encoded a polypeptide that is 300 amino acids in length. The theoretical molecular mass of the protein was $32.21 \mathrm{kDa}$, and the theoretical isoelectric point (pI) was 6.34. A putative nuclear localization signal sequence was also identified at amino acid residues 274 284 (Fig. 1 Suppl.). Sequence analysis via the InterProScan program showed that OsIAA18 protein contained an Aux/ IAA protein domain (Fig. 1 Suppl.).

A BLAST search indicated that the amino acid sequence of OsIAA18 showed a high amino acid identity with the predicted protein products of Setaria italica (XP_004951397, 67.75 \%), Brachypodium distachyon (XP- 003571137, 64.47 \%), Zea mays (ADX60088, $59 . \overline{3} \%$ ), Solanum tuberosum (XP_006343143, $42.07 \%$ ), Brassica napus (XP_013655539, $41.14 \%$ ), Cucumis sativus (XP 004150768, 40.61\%), Cucumis melo (XP_008447559, $38.79 \%$ ), Camelina sativa (XP_010504392, $38.78 \%$ ), Nicotiana tomentosiformis (XP-009628748, 38.26\%), Glycine $\max (\mathrm{XP} 003543621$, $38.22 \%$ ), Populus trichocarpa (XP_002298246, 37.90\%), Arabidopsis thaliana (AAM65282, $37.62 \%$ ), and Gossypium raimondii (XP_012445590, 36.24 \%) (Fig. 1 Suppl.). Phylogenetic analyses revealed that OsIAA18 had a close relationship with the predicted protein products of S. italica (Fig. 1 Suppl.).

To provide further evidence for the potential role of OsIAA18 in transcriptional regulation, we examined subcellular localization of OsIAA18 in onion epidermal cells. The GFP fluorescence of OsIAA18-GFP was exclusively located in the nuclei of the cells, whereas the GFP control was distributed throughout the whole onion cells (Fig. 1). These results indicate that OsIAA18 is a nuclear-localized protein.

To test whether OsIAA18 has transcription activity, OsIAA18 was fused in-frame to GAL4 DNA-binding domain in the pGBKT7 vector and the fusion constructs pBD-OsIAA18 were transformed into the yeast strain AH109. The results showed that yeast transformants harbouring pGAL4 and pBD-OsIAA18 grew normally on $\mathrm{SD} / \mathrm{Trp}^{-} / \mathrm{His}^{-}$medium exclusively and exhibited the activity of $\beta$-galactosidase reporter gene upon addition of X-gal on Whatman filter paper (Fig. 2 Suppl.). Thus these results confirm that OsIAA18 is a transcription activator.

The ORF of OSIAA18 was ectopically expressed in Arabidopsis (Col-0, WT) using the binary vector pCAMBIA1301-OsIAA18 (Fig. 2A). Multiple lines were obtained from Hyg resistance selection. Two independent

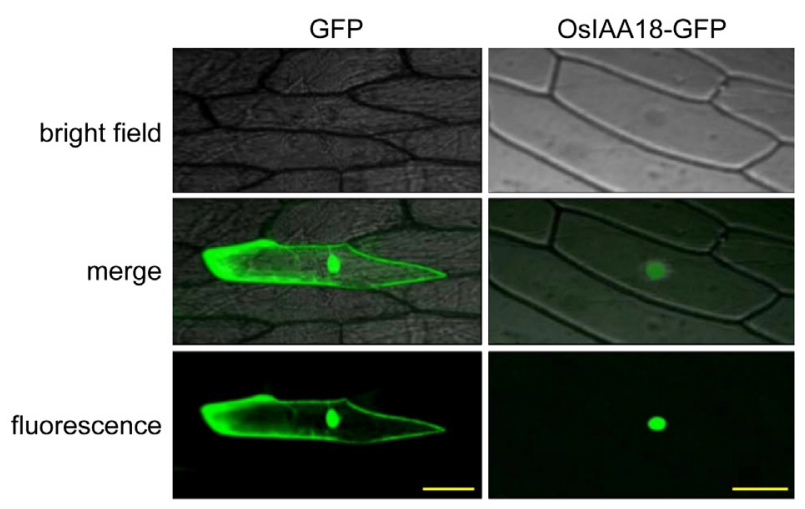

Fig. 1. Subcellular localization of the OsIAA18 protein in onion epidermal cells. The OsIAA18-GFP fusion protein was localized to the nucleus. Scale bars are $100 \mu \mathrm{m}$.

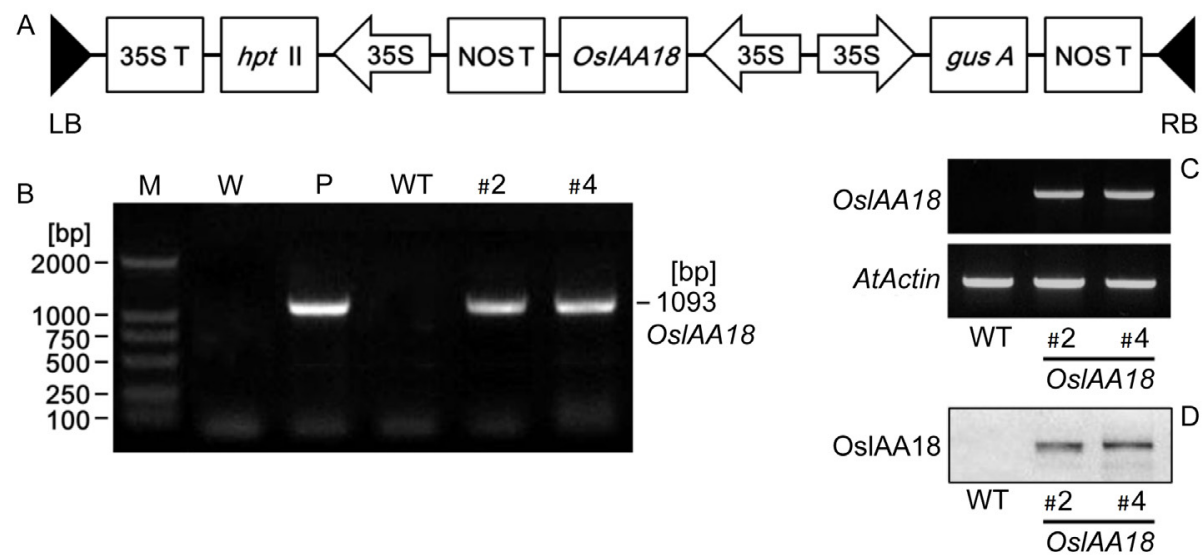

Fig. 2. Molecular analyses of the OsIAA18-expressing Arabidopsis plants. A - Aschematic diagram of the T-DNA region of the binary plasmid pCAMBIA1301-OsIAA18. LB - left border, RB - right border, hpt II - hygromycin phosphotransferase II gene, OsIAA18 - rice Aux/IAA transcription factor gene, gus $A$ - $\beta$-glucuronidase gene, $35 \mathrm{~S}$ - cauliflower mosaic virus (CaMV) $35 \mathrm{~S}$ promoter, $35 \mathrm{~S} \mathrm{~T}$ - CaMV $35 \mathrm{~S}$ terminator, NOS T - nopaline synthase terminator. $B$ - Analysis of transgenic plants by PCR. Lane $M$ - DL2000 DNA marker, lane $W$ - water as a negative control, lane $P$ - plasmid pCAMBIA1301-OsIAA18 as a positive control, lane WT - wild type, lanes \#2 and \#4 - transgenic plants. C - Expression of OSIAA18 in the OsIAA18-expressing Arabidopsis plants. The Arabidopsis AtActin gene was used as an internal control. D - Protein content of OsIAA18 in the OsIAA18-expressing Arabidopsis plants. Total proteins of $60 \mu \mathrm{g}$ from leaves were used for immunobot analysis. 
transgenic lines of Arabidopsis expressing OsIAA18 gene were obtained ( $\mathrm{T}_{1}$ generation), named $\# 2$ and \#4, and their progenies $\left(\mathrm{T}_{3}\right.$ generation) were generated. PCR analysis of genomic DNA indicated that they were transgenic (Fig. 2B). These transgenic plants were further confirmed to reveal enhanced expression of OSIAA18 gene by RTPCR and immunoblot analyses (Fig. $2 C, D$ ), thus they were selected for further experiments.

The two transgenic lines (\#2 and \#4) and WT seedlings were cultured on MS medium supplemented with $200 \mathrm{mM}$ $\mathrm{NaCl}$ and $25 \%$ PEG6000 for 2 weeks, respectively. The transgenic plants exhibited significantly higher root length and fresh mass than WT under salt and osmotic stresses, while no differences in root growth were observed under optimum conditions (Fig. 3).

The two-week-old transgenic plants (\#2 and \#4) and WT were grown in pots under $300 \mathrm{mM} \mathrm{NaCl}$ stress or disruption of watering for further evaluation of their salt and osmotic tolerance. No differences in growth were observed between the transgenic plants and WT under optimum growth condition (Fig. 4). After 4 weeks of $300 \mathrm{mM} \mathrm{NaCl}$ stress or 6 weeks after disruption of watering, the transgenic plants showed good growth, while WT died (Fig. 4). These results demonstrated that the transgenic lines (\#2 and \#4) had significantly higher salt and osmotic tolerance compared to WT.

Expression of OSIAA18, ABA biosynthesis, proline biosynthesis, stress responses, and ROS scavenging genes in the transgenic (\#2 and \#4) and WT plants were analyzed by real time qPCR. The results showed that the expression of $O S I A A 18$ was significantly higher in the transgenic plants compared to WT (Fig. 5). Under salt and osmotic stresses, the expressions of well-known salt and osmotic stress-responsive genes encoding zeaxanthin epoxidase $(A t Z E P)$, 9-cis-epoxycarotenoid dioxygenase (AtNCED), xanthoxin dehydrogenase (AtABA2), aldehyde oxidase (AtAAO), pyrroline-5-carboxylate synthase (AtP5CS), pyrroline-5-carboxylate reductase $(A t P 5 C R)$, and late embryogenesis abundant protein (AtLEA) were also higher in transgenic plants (Fig. 5). Up-regulation of the reactive oxygen species (ROS) scavenging associated genes (AtSOD and AtPOD) was observed (Fig. 5). The results indicated that $O S I A A 18$ might be involved in multiple regulatory pathways.
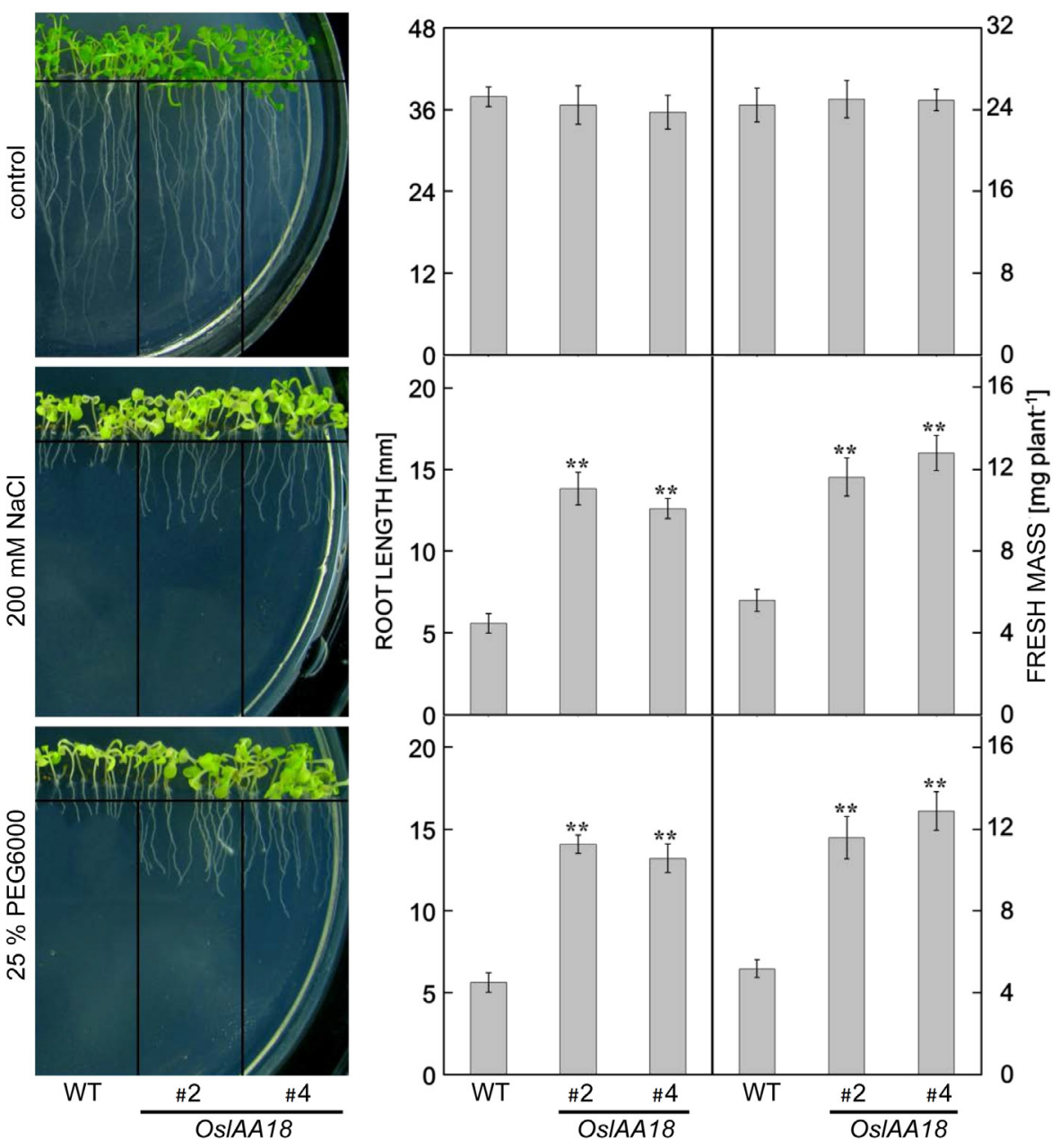

Fig. 3. Responses of transgenic and wild type Arabidopsis seedlings cultured on a Murashige and Skoog (MS) medium to salt and osmotic stresses. Arabidopsis seedlings were grown for 2 weeks on a pure MS medium (no stress), $200 \mathrm{mM} \mathrm{NaCl,} \mathrm{or} 25 \%$ (m/v) PEG6000. Root length and fresh mass were measured. Means \pm SEs, $n=3, *$ and $* *$ indicate significant differences at $P<0.05$ and $<0.01$, respectively, determined by the Student's $t$-test. 
Abscisic acid and proline, as important physiological indices, play pivotal roles in evaluating the plant stress response. ABA and proline content in the transgenic Arabidopsis plants and WT grown in pots and incubated for 4 weeks under optimum growth condition, for 2 weeks under $300 \mathrm{mM} \mathrm{NaCl}$ stress or for 4 weeks under $25 \%$ PEG6000 were analyzed. These results showed that ABA and proline content more increased in the transgenic plants compared to WT under salt and osmotic stresses (Fig. 6). Further we found that activities of NCED and P5CS (key rate limiting enzymes in $\mathrm{ABA}$ and proline biosynthesis pathways) were significantly higher in the transgenic plants compared to WT (Fig. 7).

The effects of salt and osmotic stresses on plant $\mathrm{H}_{2} \mathrm{O}_{2}$ content were examined in OsIAA18-expressing plants and WT. The results showed that the transgenic plants had significantly lower $\mathrm{H}_{2} \mathrm{O}_{2}$ content compared to WT under salt and osmotic stresses (Fig. 6). We also found that MDA accumulation was significantly decreased in the transgenic plants (Fig. 6). Meanwhile, we prompted to assay the major antioxidant enzyme (SOD and POD) activities in the transgenic and WT plants under salt and osmotic stresses. Under these stresses, the SOD and POD activities
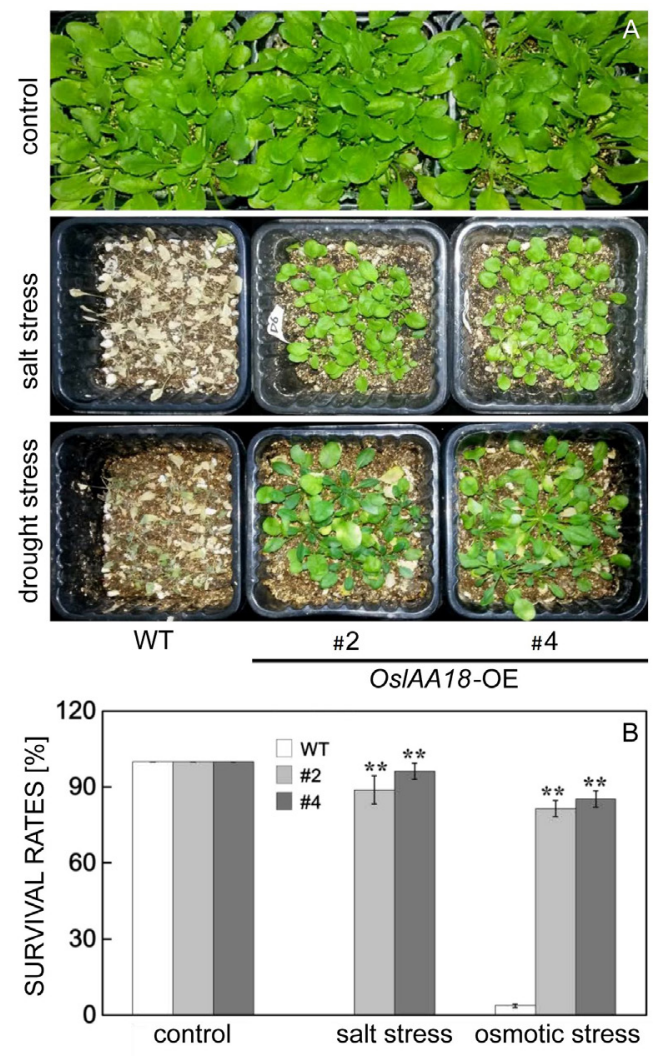

Fig. 4. Responses of the transgenic and wild type (WT) Arabidopsis plants grown in pots under salt and osmotic stresses. $A$ - Transgenic and WT plants were grown in pots and incubated for 6 weeks under optimum growth conditions, for 4 weeks under $300 \mathrm{mM} \mathrm{NaCl}$ stress, and for 6 weeks under disruption of watering. $B$ - Survival rates of transgenic and WT plants grown under salt and drought stresses. Means \pm SEs, $n=3$, * and $* *$ indicate significant differences at $P<0.05$ and $<0.01$, respectively (the Student's $t$-test). were significantly higher in the transgenic plants than in WT (Fig. 7). Thus, it is thought that overexpression of OSIAA18 reduced the accumulation of $\mathrm{H}_{2} \mathrm{O}_{2}$ and MDA and enhanced the activities of antioxidant enzymes leading to the inhibited ROS-induced damage under salt and osmotic stresses.

Electrolyte leakage measurements were performed to monitor membrane integrity. The transgenic plants had significantly lower electrolyte leakage rates compared to WT under $\mathrm{NaCl}$ and PEG treatments, whereas no significant differences in electrolyte leakage rates were observed among these plants under optimum growth condition (Fig. 8). Further analysis found that the transgenic plants had lower water loss rates compared to WT under dehydration for 2 to $6 \mathrm{~h}$ (Fig. 8). These results demonstrate some impact of the OSIAA18 gene on the conservation of water under dehydration.

\section{Discussion}

The OSIAA genes play important roles in the developmental processes, stresses, and responses to phytohormones (Song et al. 2009, Song and Xu 2013). It was found that OsIAA18 gene was induced by salt and osmotic stresses in rice (Song et al. 2009). However, little is known about the regulatory functions of this gene. In this paper, we cloned the OSIAA18 gene from rice. The OsIAA18 protein was localized in the nucleus (Fig. 1). Further transcriptional activation analysis found that the OsIAA18 protein was a transcription activator (Fig. 2 Suppl.). Constitutive expression of OSIAA18 significantly enhanced salt and osmotic tolerance in transgenic Arabidopsis plants (Figs. 3, 4).

The study of Song et al. (2009) revealed that the OsIAA18 gene is involved in plants responses mediated by ABA. In this study, ABA content increased more in the OsIAA18-expressing Arabidopsis plants than in WT under salt and osmotic stresses (Fig. 6). Consistent with this phenomenon, we found the systematic up-regulation of the genes (AtZEP, AtNCED, AtABA2, and AtAAO) and a significant increase in activity of rate limiting enzyme (NCED) involved in ABA biosynthesis pathway in the transgenic plants under salt and osmotic stresses (Figs. 5,7 ). Thus, it is suggested that constitutive expression of OsIAA18 enhances salt and osmotic tolerance due to the up-regulation of genes involved in ABA biosynthesis, which increase the production of signalling molecules and further the expression of tolerance-responsive genes (Fig. 9).

Abscisic acid is a prime mediator of plant responses to abiotic stresses (such as salt, osmotic and cold stresses) and regulates the expression of abiotic stress-responsive genes (P5CS, P5CR, and the late embryogenesis abundant) in several plant species (Zhu 2002, Silva-Ortega et al. 2008, Dalal et al. 2009, Sripinyowanich et al. 2013, Zhai et al. 2016). In the present study, the tolerance-responsive genes mentioned above were significantly up-regulated in the OsIAA18-expressing Arabidopsis plants under salt and osmotic stresses (Fig. 5). Furthermore, the transgenic 
plants had higher amount of P5CS protein under salt and osmotic stresses (Fig. 7). Our results support that high ABA content up-regulate these tolerance-responsive genes, leading to the enhanced salt and osmotic tolerance in the OsIAA18-expressing Arabidopsis plants (Fig. 9).

In plants, the up-regulation of P5CS and P5CR expression has been shown to increase proline content, resulting in the enhanced salt and osmotic tolerance (Krasensky and Jonak 2012, Liu et al. 2014, Zhai et al. 2016). Meanwhile, the OSIAA18-expressing Arabidopsis plants had significantly higher proline content compared to WT under salt and osmotic stresses (Fig. 6). Enhanced proline accumulation might maintain osmotic balance between the intracellular and extracellular environment and protect membrane integrity, resulting in enhanced salt and osmotic tolerance. Similar results were reported in some other studies (Zhang et al. 2012, Liu et al. 2014, 2015a, Zhai et al. 2016).

Salt and osmotic stresses induce ROS production in plant cells. It is important to maintain a stronger ROS-scavenging ability under salt stress to alleviate the induced oxidative damage, especially in plant leaves where photosynthesis is dramatically impacted (Gill and Tuteja 2010). In the present study, the OsIAA18overexpressing Arabidopsis plants had significantly lower $\mathrm{H}_{2} \mathrm{O}_{2}$ accumulation compared to WT under salt and

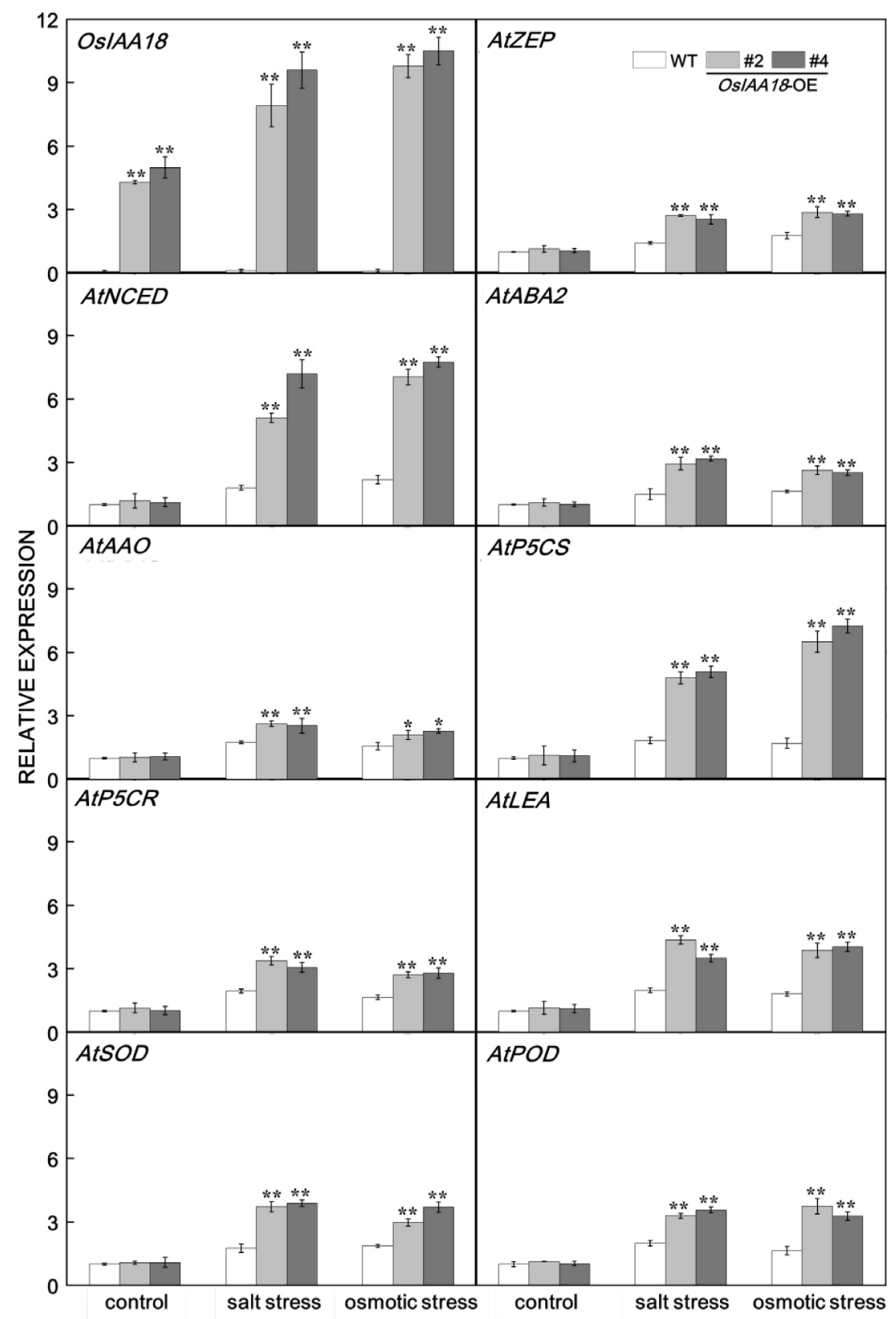

Fig. 5. Relative expressions of OSIAA18 and its related genes in the leaves of transgenic and wild type Arabidopsis plants. Pot-grown plants were incubated for 4 weeks under optimum growth conditions, for 2 weeks under $300 \mathrm{mM} \mathrm{NaCl}$, or for 4 weeks under $25 \%$ (m/v) PEG6000. The Arabidopsis actin gene was used as an internal control. Means \pm SEs, $n=3, *$ and $* *$ indicate significant differences at $P<0.05$ and $<0.01$, respectively (the Student's $t$-test). 


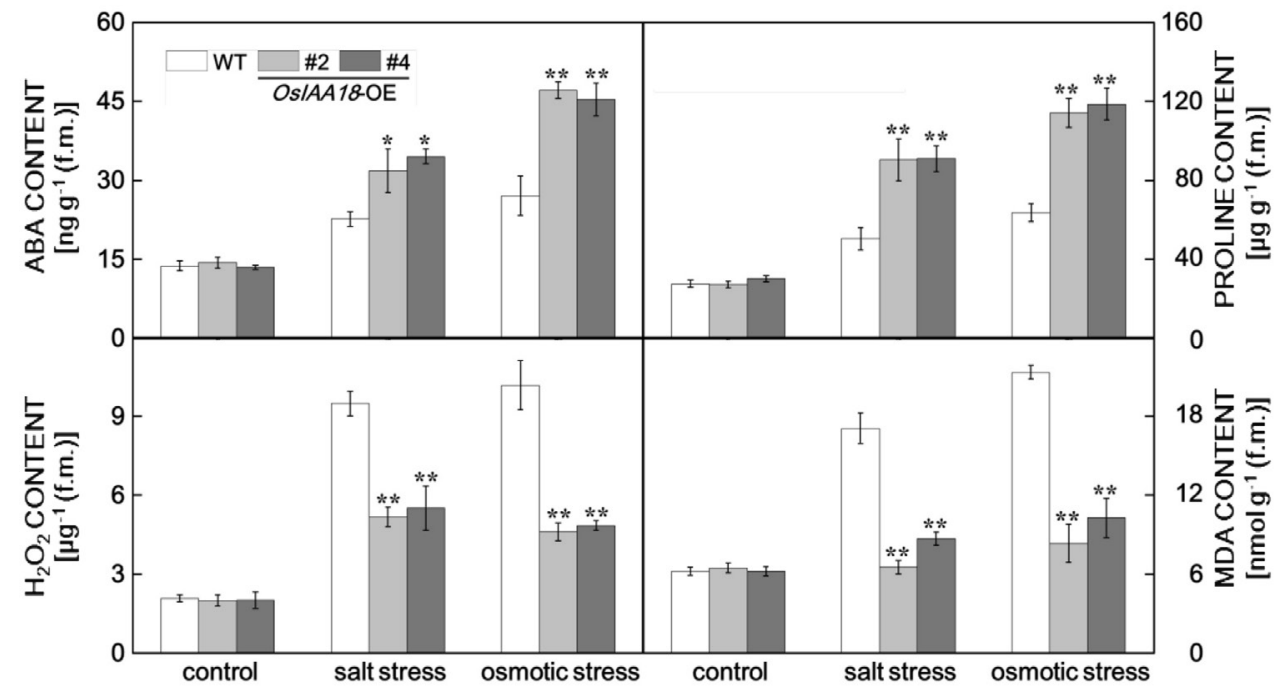

Fig. 6. The content of abscisic acid (ABA), proline, $\mathrm{H}_{2} \mathrm{O}_{2}$, and malondialdehyde (MDA) in the transgenic and wild typeArabidopsis plants. Plants grown in pots were incubated for 4 weeks under optimum growth conditions, for 2 weeks under $300 \mathrm{mM} \mathrm{NaCl}$, or for 4 weeks under $25 \%(\mathrm{~m} / \mathrm{v})$ PEG6000. Means \pm SEs, $n=3, *$ and $* *$ indicate significant differences at $P<0.05$ and $<0.01$, respectively (the Student's $t$-test).

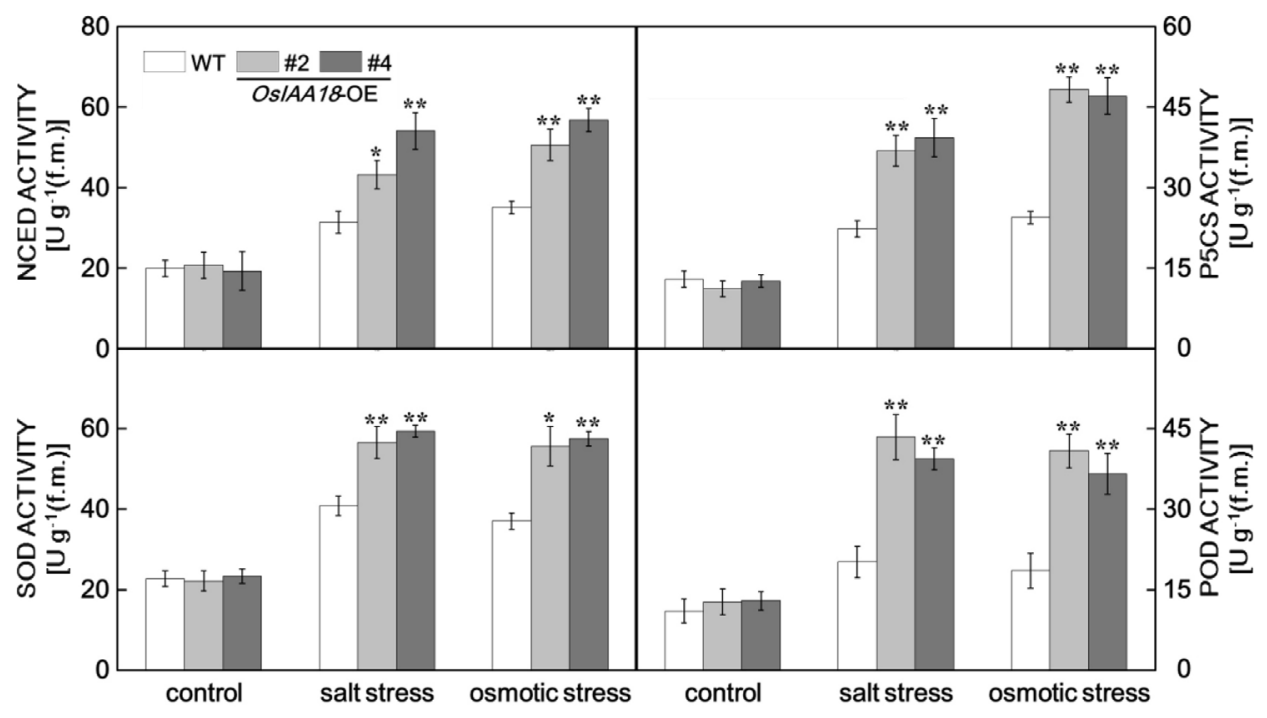

Fig. 7. The activities of 9-cis-epoxycarotenoid dioxygenase (NCED), pyrroline-5-carboxylate synthase (P5CS), superoxide dismutase (SOD), and peroxidase (POD) in the transgenic and wild type Arabidopsis plants. Plants grown in pots were incubated for 4 weeks under optimum growth conditions, for 2 weeks under $300 \mathrm{mM} \mathrm{NaCl}$, or for 4 weeks under $25 \%$ (m/v) PEG6000. Means $\pm \mathrm{SEs}, n=3$, * and $* *$ indicate significant differences at $P<0.05$ and $<0.01$, respectively (the Student's $t$-test).

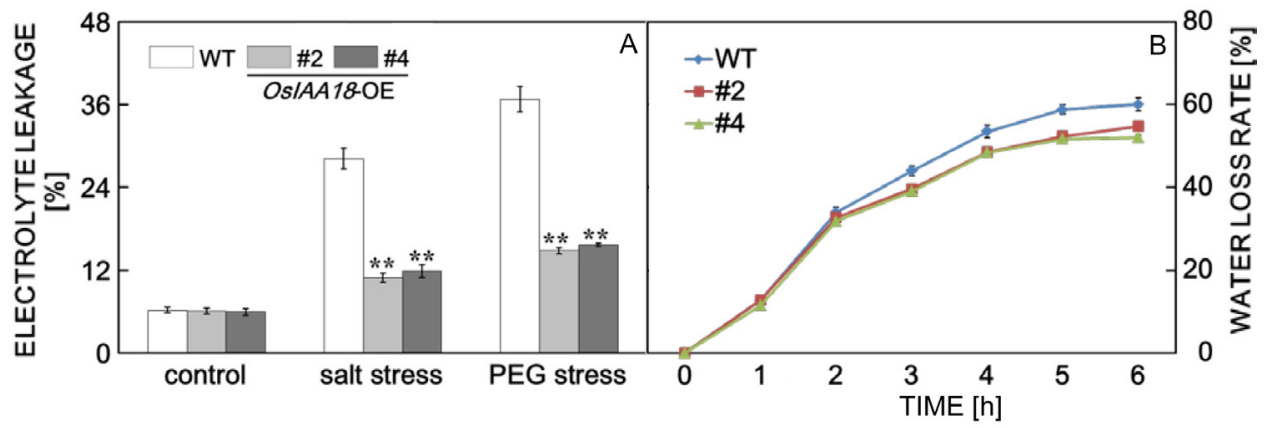

Fig. 8. Detection of electrolyte leakage and water loss rate. $A$ - Comparison of electrolyte leakage rates in in transgenic Arabidopsis and wild type (WT) plants. Plants grown in pots were treated with water (control), $300 \mathrm{mM} \mathrm{NaCl}$, and $25 \%$ PEG6000 for $48 \mathrm{~h}$. $B$ - Analysis of water loss rates in WT and transgenic plants grown under control conditions. The $x$-axis shows the designated time length for plant desiccation. Means \pm SEs, $n=3, *$ and ** indicate significant differences at $P<0.05$ and $<0.01$, respectively (the Student's $t$-test). 


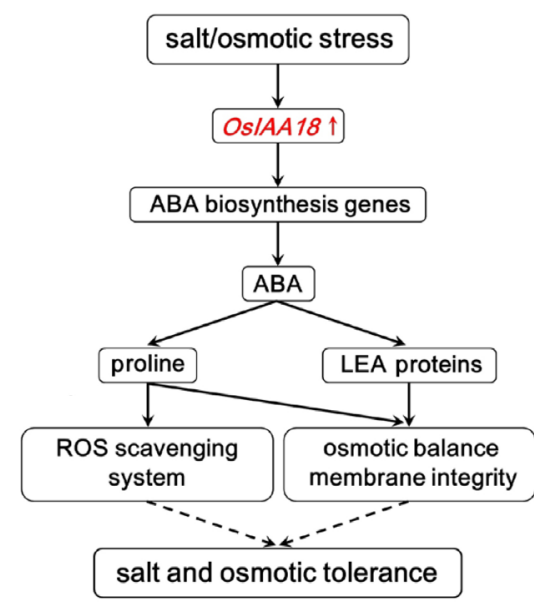

Fig. 9. The hypothesis of the regulatory network of the OsIAA18 gene involved in salt and osmotic stresses responses. Constitutive expression of OSIAA18 up-regulates genes involved in abscisic acid (ABA) biosynthesis, proline biosynthesis, and reactive oxygen species (ROS) scavenging, which results in significant physiological changes, including increased $\mathrm{ABA}$ and proline content and reduced ROS accumulation, leading to an enhanced salt and osmotic tolerance. LEA - late embryogenesis abundant.

osmotic stresses (Fig. 6). We also found that the systematic up-regulation of ROS scavenging genes (AtSOD and $A t P O D)$ and a significant increase of antioxidant enzyme (SOD and POD) activities were observed in the transgenic plants under salt and osmotic stresses (Figs. 5, 7). Therefore, improved salt and osmotic tolerance of the transgenic plants might be due, at least in part, to the enhanced ROS scavenging capacity (Zhang et al. 2012, Liu et al. 2014, 2015a, Zhai et al. 2016, Wang et al. 2018). In addition, proline is an effective scavenger of singlet oxygen and hydroxyl radicals (Alia et al. 2001, Smirnoff and Cumbes 1989). Our results support that enhanced proline accumulation activates ROS scavenging system, leading to the enhanced salt and osmotic tolerance in the OsIAA18-expressing Arabidopsis plants (Liu et al. 2014, 2015a, Zhai et al. 2016, Wang et al. 2018) (Fig. 9).

In addition, late embryogenesis abundant protein, as one of the most important stress-associated gene families, plays pivotal roles in stress tolerance, as osmotic adjustment material, as a protection material for cell membrane structure (Dalal et al. 2009, Jia et al. 2014, Banerjee et al. 2015). The late embryogenesis abundant genes have been shown to enhance tolerance to salt and osmotic stresses in plants (Park et al. 2011, Zhao et al. 2011, Ganguly et al. 2012, Muñoz-Mayor et al. 2012, Gao et al. 2013). In the present study, the expression of AtLEA gene was upregulated in the OSIAA18-expressing Arabidopsis plants under salt and osmotic stresses, indicating the marked improvement of their salt and osmotic tolerance (Figs. 5 and 9).

\section{Conclusions}

In this study, OsIAA18 gene has been successfully cloned from rice. Constitutive expression of OSIAA18 significantly enhanced salt and osmotic tolerance in transgenic Arabidopsis plants. Our results suggest that the OsIAA18 gene may enhance salt and osmotic tolerance of Arabidopsis transgenic plants by up-regulating the expression of ABA biosynthesis, proline biosynthesis, stress responses, and ROS scavenging genes. OsIAA18 gene might be a hopeful candidate for enhancing tolerance to abiotic stresses also in other plants.

\section{References}

Alexieva, V., Sergiev, I., Mapelli, S., Karanov, E.: The effect of osmotic and ultraviolet radiation on growth and stress markers in pea and wheat. - Plant Cell Environ. 24: 1337-1344, 2001.

Alia, Mohanty, P, Matysik, J.: Effect of proline on the production of singlet oxygen. - Amino Acids 21: 195-200, 2001.

Banerjee, A., Roychoudhury, A.: Group II late embryogenesis abundant (LEA) proteins: structural and functional aspects in plant abiotic stress. - Plant Growth Regul. 79: 1-17, 2015.

Ben-Amor, M., Flores, B., Latche, A., Bouzayen, M., Pech, J.C., Romojaro, F.: Inhibition of ethylene biosynthesis by antisense ACC oxidase RNA prevents chilling injury in Charentais cantaloupe melons. - Plant Cell Environ. 22: 1579-1586, 1999.

Berleth, T., Krogan, N.T., Scarpella, E.: Auxin signals-turning genes on and turning cells around. - Curr. Opin. Plant Biol. 7: 553-563, 2004.

Bohnert, H.J., Nelson, D.E., Jensen, R.G.: Adaptations to environmental stresses. - Plant Cell 7: 1099-1111, 1995.

Dalal, M., Tayal, D., Chinnusamy, V., Bansal, K.C.: Abiotic stress and ABA-inducible group 4 LEA from Brassica napus plays a key role in salt and osmotic tolerance. - J. Biotechnol. 139: 137-145, 2009.

Friml, J.: Auxin transport-shaping the plant. - Curr. Opin. Plant Biol. 6: 7-12, 2003.

Ganguly, M., Datta, K., Roychoudhury, A., Gayen, D., Sengupta, D.N., Datta, S.K.: Overexpression of Rab16A gene in indica rice variety for generating enhanced salt tolerance. - Plant Signal. Behav. 7: 502-509, 2012.

Gao, S., Yu, B., Yuan, L., Zhai, H., He, S.Z., Liu, Q.C.: Production of transgenic sweet potato plants resistant to stem nematodes using oryzacystatin-I gene. - Sci. Hort. 128: 408-414, 2011 a.

Gao, S., Yuan, L., Zhai, H., Liu, C.L., He, S.Z., Liu, Q.C.: Transgenic sweetpotato plants expressing an LOS5 gene are tolerant to salt stress. - Plant Cell Tissue Organ Cult. 107: 205-213, 2011b.

Gao, W.D., Bai, S., Li, Q.M., Gao, C.Q., Liu, G.F., Li, G.D., Tan, F.L.: Overexpression of TaLEA gene from Tamarix androssowii improves salt and osmotic tolerance in transgenic poplar (Populus simonii $\times$ P. nigra). - PLoS ONE 8: e67462, 2013.

Gill, S.S., Tuteja, N.: Reactive oxygen species and antioxidant machinery in abiotic stress tolerance in crop plants. - Plant Physiol. Biochem. 48: 909-930, 2010.

Hagen, G., Guilfoyle, T.: Auxin-responsive gene expression: genes, promoters and regulatory factors. - Plant mol. Biol. 49: 373-385, 2002.

Hayzer, D.J., Leisinger, T.: The gene-enzyme relationships of proline biosynthesis in Escherichia coli. - J. gen. Microbiol. 118: 287-293, 1980.

Jain, M., Kaur, N., Garg, R., Thakur, J.K., Tyagi, A.K., Khurana, J.P.: Structure and expression analysis of early auxinresponsive Aux/IAA gene family in rice (Oryza sativa). Funct. integr. Genomics 6: 47-59, 2006. 
Jefferson, R.A., Kavanagh, T.A., Bevan, M.W.: GUS fusions: $\beta$-glucuronidase as a sensitive and versatile gene fusion marker in higher plants. - EMBO J. 6: 3901-3907, 1987.

Jia, F., Qi, S., Li, H., Liu, P., Li, P., Wu, C., Zheng, C., Huang, J.: Overexpression of late embryogenesis abundant 14 enhances Arabidopsis salt stress tolerance. - Biochem. Biophys. Res. Commun. 454: 505-511, 2014.

Jiang, X., Zhang, C., Lü, P., Jiang, G., Liu, X., Dai, F., Gao, J.: RhNAC3, a stress-associated NAC transcription factor, has a role in dehydration tolerance through regulating osmotic stress-related genes in rose petals. - Plant Biotechnol. J. 12: 38-48, 2014.

Ni, J., Wang, G.H., Zhu, G.H., Zhang, H.H., Wu, Y.R., Wu, P.: OsIAA23-mediated auxin signaling defines postembryonic maintenance of QC in rice. - Plant J. 68: 433-442, 2011.

Kitomi, Y., Inahashi, H., Takehisa, H., Sato, Y., Inukai, Y.: OsIAA13-mediated auxin signaling is involved in lateral root initiation in rice. - Plant Sci. 190: 116-122, 2012.

Krasensky, J., Jonak, C.: Osmotic, salt, and temperature stressinduced metabolic rearrangements and regulatory networks. - J. exp. Bot. 63: 1593-1608, 2012.

Li, X., Ma, H., Huang, H., Li, D., Yao, S.: Natural anthocyanins from phytoresources and their chemical researches. - Nat. Prod. Res. 27: 456-469, 2013.

Liscum, E., Reed, J.W.: Genetics of Aux/IAA and ARF action in plant growth and development. - Plant mol. Biol. 49: 387 400, 2002.

Liu, D.G., He, S.Z., Song, X.J., Zhai, H., Liu, N., Zhang, D.D., Ren, Z.T., Liu, Q.C.: IbSIMT1, a novel salt-induced methyltransferase gene from Ipomoea batatas, is involved in salt tolerance. - Plant Cell Tissue Organ Cult. 120: 701-715, 2015a.

Liu, D.G., He, S.Z., Zhai, H., Wang, L.J., Zhao, Y., Wang, B., Li, R.J., Liu, Q.C.: Overexpression of IbP5CR enhances salt tolerance in transgenic sweetpotato. - Plant Cell Tissue Organ Cult. 117: 1-16, 2014.

Liu, Y., Ji, X., Nie, X., Qu, M., Zheng, L., Tan, Z., Zhao, H., Huo, L., Liu, S., Zhang, B., Wang, Y.: Arabidopsis AtbHLH112 regulates the expression of genes involved in abiotic stress tolerance by binding to their E-box and GCG-box motifs. New Phytol. 207: 692-709, 2015b.

Lou, X.M., Yao, Q.H., Zhang, Z., Peng, R.H., Xiong, A.S., Wang, K.K.: Expression of human hepatitis B virus large surface antigen gene in transgenic tomato. - Clin. Vaccine Immunol. 14: 464-469, 2007.

Lurie, S., Fallika, E., Handrosa, A., Shapirab, R.: The possible involvement of peroxidase in resistance to Botrytis cinerea in heat treated tomato fruit. - Physiol. mol. Plant. Pathol. 50: 141-149, 1997.

Munns, R., Tester, M.: Mechanisms of salinity tolerance. Annu. Rev. Plant Biol. 59: 651-681, 2008.

Muñoz-Mayor, A., Pineda, B., Garcia-Abellán, J.O., Antón, T., Garcia-sogo, B., Sanchez-Bel, P., Flores, F.B., Atarés, A., Angosto, T., Pintor-Toro, J.A., Moreno, V., Bolarin, M.C.: Overexpression of dehydrin tas 14 gene improves the osmotic stress imposed by osmotic and salinity in tomato. - J. Plant Physiol. 169: 459-468, 2012.

Murashige, T., Skoog, F.: A revised medium for rapid growth and bioassays with tobacco tissue cultures. - Physiol. Plant 15: 473-497, 1962.

Nakamura, A., Umemura, I., Gomi, K., Hasegawa, Y., Kitano, H., Sazuka, T., Matsuoka, M.: Production and characterization of auxininsensitive rice by overexpression of a mutagenized rice IAA protein. - Plant J. 46: 297-306, 2006.

Park, S.C., Kim, Y.H., Jeong, J.C., Kim, C.Y., Lee, H.S., Bang, J.W., Kwak, S.S.: Sweet potato late embryogenesis abundant
14 (IbLEA14) gene influences lignification and increases osmotic- and salt stress-tolerance of transgenic calli. - Planta 233: 621-634, 2011.

Reed, J.W.: Roles and activities of Aux/IAA proteins in Arabidopsis. - Trends Plant Sci. 6: 420-425, 2001.

Schmittgen, T.D., Livak, K.J.: Analyzing real-time PCR data by the comparative $\mathrm{C}_{\mathrm{T}}$ method. - Nat. Protocols 3: 1101-1108, 2008.

Silva-Ortega, C.O., Ochoa-Alfaro, A.E., Reyes-Agüero, J.A., Aguado-Santacruz, G., Jiménez-Bremont, J.F.: Salt stress increases the expression of $p 5 c s$ gene and induces proline accumulation in cactus pear. - Plant Physiol. Biochem. 46: 82-92, 2008 .

Smirnoff, N., Cumbes, Q.J.: Hydroxyl radical scavenging activity of compatible solutes. - Phytochemistry 28: 1057-1060, 1989.

Song, Y., Wang, L., Xiong, L.: Comprehensive expression profiling analysis of OSIAA gene family in developmental processes and in response to phytohormone and stress treatments. - Planta 229: 577-591, 2009.

Song, Y.L., Xu, Z.F.: Ectopic overexpression of an auxin/ indole-3-acetic acid (Aux/IAA) gene OsIAA4 in rice induces morphological changes and reduces responsiveness to auxin. - Int. J. mol. Sci. 14: 13645-13656, 2013.

Sripinyowanich, S., Klomsakul, P., Boonburapong, B., Bangyeekhun, T., Asami, T., Gu, H., Buaboocha, T., Chadchawan, S.: Exogenous ABA induces salt tolerance in indica rice (Oryza sativa L.): The role of OsP5CS1 and OSP5CR gene expression during salt stress. - Environ. exp. Bot. 86: 94-105, 2013.

Thakur, J.K., Jain, M., Tyagi, A.K., Khurana, J.P.: Exogenous auxin enhances the degradation of a light down-regulated and nuclear localized OsiIAA1, an Aux/IAA protein from rice, via proteasome. - Biochim. biophys. Acta. 1730: 196-205, 2005.

Thakur, J.K., Tyagi, A.K., Khurana, J.P.: OsIAA1, an Aux/IAA cDNA from rice, and changes in its expression as influenced by auxin and light. - DNA Res. 8: 193-203, 2001.

Vogler, H., Kuhlemeier, C.: Simple hormones but complex signalling. - Curr. Opin. Plant Biol. 6: 51-56, 2003.

Wang, D., Pei, K., Fu, Y., Sun, Z., Li, S., Liu, H., Tang, K., Han, B., Tao, Y.: Genome-wide analysis of the auxin response factors $(A R F)$ gene family in rice (Oryza sativa). - Gene 394: 13-24, 2007.

Wang, F.B., Kong, W.L., Wong, G., Fu, L.F., Peng, R.H., Li, Z.J., Yao, Q.H.: AtMYB12 regulates flavonoids accumulation and abiotic stress tolerance in transgenic Arabidopsis thaliana. Mol. Genet. Genomics 291: 1545-1559, 2016a.

Wang, F.B., Tong, W.J., Zhu, H., Kong, W.L., Peng, R.H., Liu, Q.C., Yao, Q.H. A novel $\mathrm{Cys}_{2} / \mathrm{His}_{2}$ zinc finger protein gene from sweet potato, IbZFPl, is involved in salt and osmotic tolerance in transgenic Arabidopsis. - Planta 243: 783-797, $2016 b$.

Wang, F.B., Zhu, H., Chen, D.H., Li, Z.J., Peng, R.H., Yao, Q.H.: A grape bHLH transcription factor gene, $V v b H L H 1$, increases the accumulation of flavonoids and enhances salt and osmotic tolerance in transgenic Arabidopsis thaliana. - Plant Cell Tissue Organ Cult. 125: 387-398, 2016 c.

Wang, F.B., Zhu, H., Kong, W.L., Peng, R.H., Liu, Q.C., Yao, Q.H.: The Antirrhinum AmDEL gene enhances flavonoids accumulation and salt and osmotic tolerance in transgenic Arabidopsis. - Planta 244: 59-73, 2016 d.

Wang, F.B., Ren, G.L., Li, F.S., Qi, S.T., Xu, Y., Wang, B.W., Yang, Y.L., Ye, Y.X., Zhou, Q., Chen, X.H.: A chalcone synthase gene AeCHS from Abelmoschus esculentus regulates flavonoids accumulation and abiotic stress tolerance in transgenic Arabidopsis. - Acta Physiol. Plant. 40: 97, 2018.

Wang, G.L., Fang, H.Y.: Plant Genetic Engineering. - Science 
Press, Beijing 2002.

Yamamoto, K.T.: Further characterization of auxin-regulated mRNAs in hypocotyl sections of mung bean [Vigna radiata (L.) Wilczek]: sequence homology to genes for fatty-acid desaturases and atypical late-embryogenesis-abundant protein, and the mode of expression of the mRNAs. - Planta 192: 359-364, 1994

Yang, S.J, Vanderbeld, B., Wan, J.X., Huang, Y.F.: Narrowing down the targets: towards successful genetic engineering of osmotic-tolerant crops. - Mol. Plants 3: 469-490, 2010.

Zhai, H., Wang, F.B., Si, Z.Z., Huo, J.X., Xing, L., An, Y.Y., He, S.Z., Liu, Q.C.: A myo-inositol-1-phosphate synthase gene, IbMIPS1, enhances salt and osmotic tolerance and stem nematode resistance in transgenic sweetpotato. - Plant Biotechnol. J. 14: 592-602, 2016.

Zhang, H., Han, B., Wang, T., Chen, S., Li, H., Zhang, Y., Dai, S.: Mechanisms of plant salt response: insights from proteomics. - J. Proteome Res. 11: 49-67, 2012.
Zhang, X., Henriques, R., Lin, S.S.: Agrobacterium-mediated transformation of Arabidopsis thaliana using the floral dip method. - Nat. Protocols 1: 641-646, 2006.

Zhao, P., Liu, F., Ma, M., Gong, J., Wang, Q., Jia, P., Zheng, G., Liu, H.: Overexpression of AtLEA3-3 confers resistance to cold stress in Escherichia coli and provides enhanced osmotic stress tolerance and ABA sensitivity in Arabidopsis thaliana. - Mol. Biol. 45: 851-862, 2011.

Zhao, Q., Zhang, H., Wang, T., Chen, S.X., Dai, S.J.: Proteomicsbased investigation of salt-responsive mechanisms in plant roots. - J. Proteomics 82: 230-253, 2013.

Zhu, J.K.: Plant salt tolerance. - Trends Plant Sci. 6: 66-71, 2001. Zhu, J.K.: Salt and osmotic stress signal transduction in plants. Annu. Rev. Plant Biol. 53: 247-273, 2002.

Zhu, Z.X., Liu, Y., Liu, S.J., Mao, C.Z., Wu, Y.R., Wu, P.: A gain-of-function mutation in OSIAA11 affects lateral root development in rice. - Mol. Plants 5: 154-161, 2012. 